\title{
Childhood obesity and prevention in different socio-economic contexts
}

\author{
Maria B. Magnusson, Agneta Sjöberg, Karin I. Kjellgren and Lauren Lissner
}

\section{Linköping University Post Print}

N.B.: When citing this work, cite the original article.

Original Publication:

Maria B. Magnusson, Agneta Sjöberg, Karin I. Kjellgren and Lauren Lissner, Childhood obesity and prevention in different socio-economic contexts, 2011, Preventive Medicine, (53), 6, 402-407.

http://dx.doi.org/10.1016/j.ypmed.2011.09.019

Copyright: Elsevier

http://www.elsevier.com/

Postprint available at: Linköping University Electronic Press

http://urn.kb.se/resolve?urn=urn:nbn:se:liu:diva-73548 
Corresponding author:

Maria B Magnusson ${ }^{12}$

$\mathrm{RD}, \mathrm{RN}, \mathrm{M} \mathrm{Sc}$

Public Health Epidemiology Unit

Department of Public Health and Community Medicine

Box 454

S-405 30 Göteborg

Telephone: +46 31-786 6818

Telefax: + $4631-7781704$

e-mail: maria.magnusson@allmed.gu.se

Co-authors:

Agneta Sjöberg ${ }^{1}$

$\mathrm{PhD}$ (Medicine)

agneta.sjoberg@allmed.gu.se

Karin I Kjellgren ${ }^{3,4}$

$\mathrm{PhD}$ (Medicine)

karin.kjellgren@fhs.gu.se

Lauren Lissner ${ }^{1}$

$\mathrm{PhD}$ (Medicine)

lauren.lissner@medfak.gu.se

${ }^{1}$ Department of Public Health and Community Medicine, Public Health Epidemiology Unit, The Sahlgrenska Academy at the University of Gothenburg, Sweden

2Primary Health Care Clinic Angered, Gothenburg

${ }^{3}$ Institute of Health and Care Sciences, Sahlgrenska Academy, University of Gothenburg, Gothenburg, Sweden

${ }^{4}$ Department of Medical and Health Sciences, Division of Nursing Science, Faculty of Health Sciences, Linköping University, Linköping, Sweden 


\section{Childhood obesity and prevention in different socio-economic contexts}

\section{Abstract}

Objective: To assess recent trends in obesity, health beliefs, and lifestyles in Swedish schoolchildren, with focus on socioeconomic disparities.

Method: The study was conducted in two areas with high and low socioeconomic status (SES). 340 11-12 year olds participated in three cross-sectional surveys assessing food-related behaviours, physical activity and health beliefs, together with anthropometric examinations. Comparisons were made before and after a community-based intervention (2003 versus 2008) within the low-SES school, and between the low and high-SES school (2008 only).

Results: In the low-SES school BMI z-score decreased over 5 years $(0.80$ vs 0.46$)$ as did the percentage of children frequently consuming sweet drinks ( $43.5 \mathrm{vs} 26.8 \%$ ), statistically significant in girls only $(\mathrm{p}<0.05)$. Children increasingly perceived benefits of healthy lifestyles (37 vs $55 \%$ ). In 2008, consumption of breakfast, vegetables, sweets and sweet drinks differed between schools, as did screen-time and physical activity, all in favour of the high SES-school where the obesity-prevalence was significantly lower (0.8 vs 6.7\%).

Conclusion: Positive changes in diet and weight status were observed, especially in girls, within a low-income multi-ethnic community undergoing a health promotion intervention. Our results underscore the multifactorial etiology of childhood obesity and the importance of continuing tailored, gender-sensitive prevention efforts.

Keywords: adolescent; child; emigrants and immigrants; health behaviour; obesity; overweight; primary prevention; socioeconomic factors

\section{Introduction}

Childhood obesity is known to have socioeconomic and cultural, as well as genetic origins (Shrewsbury and Wardle 2008). Current evidence suggests a high and possibly increasing burden of obesity in low income groups, including an increased risk among ethnic minorities (de Wilde et al. 2009, Waters et al. 2008). However in Sweden the epidemic appears to be slowing down, particularly among urban girls (Sjoberg et al. 2008, Sundblom et al. 2008). Despite these encouraging trends, socioeconomic disparities remain. A variety of reasons have been suggested for the higher prevalence of obesity in lower income and certain ethnic groups, as well as for gender disparities (Kumanyika 2008a). 
Interventions against childhood obesity have often been ineffective. More knowledge is required for better understanding of how to design effective primary and secondary prevention interventions, and in particular how to work in socio-economically disadvantaged areas representing a variety of ethnic backgrounds (Kumanyika 2008b).

In January 2003 we conducted a school-based survey in children aged 11-12 years in a residential area of Gothenburg, Sweden with low SES and, compared to the rest of the city, a large proportion of immigrants and refugees (Magnusson et al. 2005). Main findings were an unexpectedly high prevalence of overweight and obesity combined (31\%) together with a high intake of calorically sweetened beverages and a low degree of perceived benefit from healthy lifestyles. In 2004, a community based intervention "Health Equilibrium Initiative" (HEI) was launched in this area with the aim of developing methods to prevent obesity and overweight among children (Fig 1). The intervention was based upon theories involving health, health promotion and culture (Eriksen 2002, Champion and Skinner 2008, McAlister et al. 2008). It employed an "upstream approach", i.e. rather than aiming exclusively at people with risk factors arenas were targeted where determinants of obesity could be addressed. Culturally sensitive methods were developed to promote healthy food choices and increased physical activity (PA), and to support self-efficacy and media-literacy. A dietician and a district nurse from primary care offered tailored information in 13 languages and inspiration for change to staff, parents and children in the broad set of community arenas targeted by the intervention. The intervention is described in more detail elsewhere (Magnusson 2011, in Appendix). In order to follow up this initiative it was decided to undertake a new survey of the corresponding population five years later. To shed light on factors behind the socioeconomic discrepancies in obesity, a survey of children in an area with high SES was carried out simultaneously. 
Figure 1 Working model of Health Equilibrium Initiative

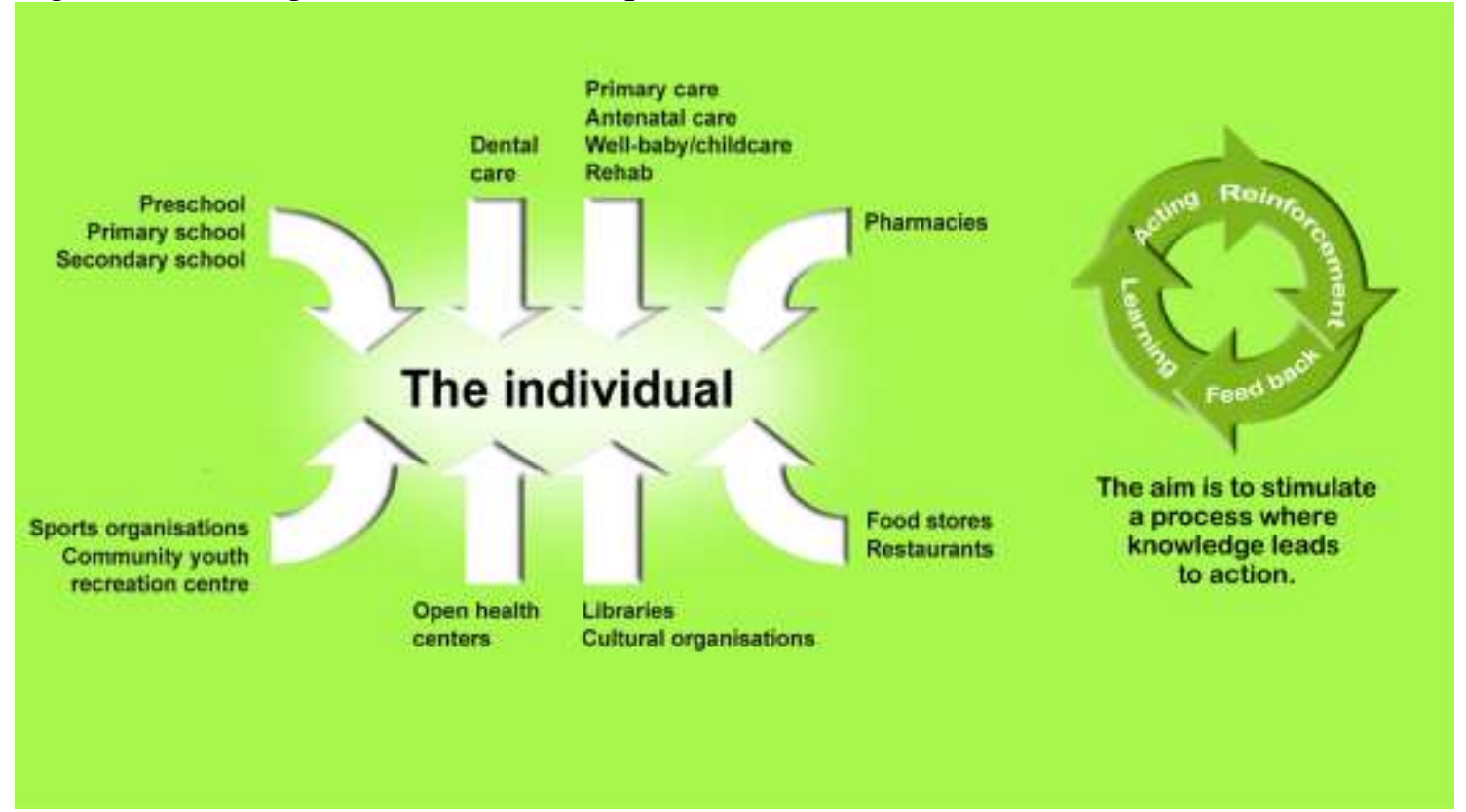

The surveys included in the study were conducted in Gothenburg, Sweden in 2003 and 2008. The aim is to help individuals and groups to develop knowledge and experience relevant to their health needs, facilitated by communication of current recommendations on diet and physical activity. The material ("Balance-leaflet") was available in 13 languages and offered in multiple settings.

This is an updated version of the Working model, published in (Magnusson 2011)

Magnusson, M. , 2011. Childhood obesity prevention in the context of socioeconomic status and migration. In Unit of Public Health Epidemiology, The University of Gothenburg, Gothenburg. http://gupea.ub.gu.se/handle/2077/23818 (downloaded 2011-07-26)

The aim of the present study was twofold: (i) to compare two cohorts of school children in a low SES area within a time-space of five years; and (ii) to compare school children in two areas of high and low SES in the more recent survey. Specifically we aim to assess the prevalence of overweight and obesity, selected food-related behaviours, indicators of PA/ inactivity and perceived benefit of a healthy lifestyle.

\section{Methods}

This was an ecological longitudinal study consisting of cross-sectional surveys carried out in 2003 and 2008. In 2003, a school in a low-SES neighbourhood was identified and all children 
in grades 5-6 (11-12 y) were recruited. Results from this survey have been reported (Magnusson et al. 2005). After 5 years, the school was re-visited and a new cohort of 11-12 year olds was examined. At the same time, children in the same age from a high-SES school were studied by the same protocol.

The results from the first survey were worrisome and inspired the launching of the community based intervention described in the background, in the low SES community. After this intervention had been in place for several years we conducted the 2008 surveys, using the same survey methods as in 2003. In both 2003 and 2008, all pupils in grades five and six, aged 11 to 12 years $(n=342)$ were invited to participate. With the purpose of elucidating habitual dietary intake, meal pattern, physical activity and perceived benefit of a healthy lifestyle, a 15 minute long, structured, individual interview was used together with a selfadministered questionnaire. The instrument was adapted from a validated questionnaire and interview, used in the Gothenburg Adolescence Study (Sjöberg et al. 2003). Following a pilot study in 2003, some of the original dietary questions were simplified for an age group with a lower degree of proficiency in Swedish, a change that was justified considering that the present aim was not to calculate nutrient intake. A more detailed description of the questionnaire and supplementary interview is presented elsewhere (Magnusson et al. 2005).

In the first survey conducted in 2003, 114 pupils were invited and 112 participated (100 were measured, 108 answered the questionnaire and were interviewed). In 2008, all the invited pupils ( $\mathrm{n}=228$ ) participated ( 226 children were measured, 218 answered the questionnaire and 216 were interviewed). The anthropometric examinations were conducted by skilled school nurses. In both surveys, body weight was measured in indoor clothes to the nearest $0.1 \mathrm{~kg}$ with standard digital scales. Height was measured without shoes to the nearest $0.01 \mathrm{~m}$. The questionnaire was administered by two researchers who co-operated to coordinate the study. They initially visited the schools together and then conducted their main part of the study on one school each, while continuously conferring on methodological standardization.

Comparisons were made in the area with low SES between 2003 vs 2008, and between the low and high SES schools in 2008. The low SES school in 2008 was used as a reference. 
Overweight and obesity were defined using cut off points according to the International Obesity Task Force (Cole et al. 2000). Thinness was defined by cut offs suggested by Cole et al (2007). The distribution of BMI z-score as compared to the normal Swedish population reference curves was calculated by the equation presented by Karlberg et al (2001) and presented as histograms. In addition to anthropometry, we have compared 15 variables as markers of lifestyle habits with recognised connections to obesity and general health (Enghardt Barbieri et al. 2006, Fogelholm 2008, Toschke et al. 2009).

Calculations were made using Statistical Package for the Social Sciences (SPSS) version 15.0. Differences between the low SES school in 2008 and the other two groups were tested by chisquare test or ANOVA, with p-levels < 0.05 considered significant. All analyses were stratified by sex and pooled with adjustment for sex.

Both surveys were approved by The Ethics Committee at the University of Gothenburg (Study codes Ö 525-02 and T 574-07). The study was conducted during school hours and all procedures and questions were such that might have been part of the regular health education. All families received written information about the study before it started.

\section{Results}

\section{ANTHROPOMETRIC TRENDS IN LOW SES SCHOOL (2003 versus 2008)}

For statistical purposes, the city of Gothenburg is divided in 94 municipalities. For this study, two such municipalities with major differences between them concerning important SES indicators were chosen. 
Table 1 Indicators of socio-economic status for the low SES area in 2003 and 2008 and for the high SES area in 2008

\begin{tabular}{|c|c|c|c|}
\hline & $\begin{array}{l}\text { Area with low } \\
\text { SES } 2003\end{array}$ & $\begin{array}{l}\text { Area with low } \\
\text { SES } 2008\end{array}$ & $\begin{array}{l}\text { Area with high } \\
\text { SES } 2008\end{array}$ \\
\hline Number of inhabitants & 6875 & 7806 & 10176 \\
\hline Surface & $2 \mathrm{~km}^{2}$ & $2 \mathrm{~km}^{2}$ & $22 \mathrm{~km}^{2}$ \\
\hline $\begin{array}{l}\text { Potential refugees (i.e. persons } \\
\text { from former Yugoslavia, Iran, } \\
\text { Iraq, Somalia) } \%\end{array}$ & 28 & 25 & 06 \\
\hline Persons $25-64$ years employed $\%$ & 47 & 51 & 89 \\
\hline Tertiary education $\%$ & 19 & 21 & 63 \\
\hline $\begin{array}{l}\text { Yearly average income (Swedish } \\
\text { crowns) }\end{array}$ & 127900 & $146900^{1}$ & 355700 \\
\hline $\begin{array}{l}\text { As compared with an index of } \\
\frac{100 \text { (for Gothenburg as a }}{\text { whole) }}\end{array}$ & & & \\
\hline $\overrightarrow{\text { Unemployed }}$ & 193 & 233 & 41 \\
\hline Tertiary education & 43 & 44 & 133 \\
\hline Average income & 62 & 62 & 154 \\
\hline High income & 12 & 27 & 214 \\
\hline
\end{tabular}

${ }^{1}$ when taking into account the inflation of the Swedish crown ( $8 \%$ over the five year interval) the mean income is $6 \%$ higher in 2008 than in 2003

The surveys included in the study were conducted in Gothenburg, Sweden in 2003 and 2008.

In the school with low SES in the intervention community, the BMI z-score distribution curve shifted over time (Figure 2) with a significant decrease for the girls but not for the boys (Table 2). The prevalence of obesity decreased non-significantly from $13 \%$ to $6.7 \%$ during the five years, similarly distributed between boys and girls. For girls, there was a significant increase in thinness grade 1-2, but not for the boys. 
Figure 2
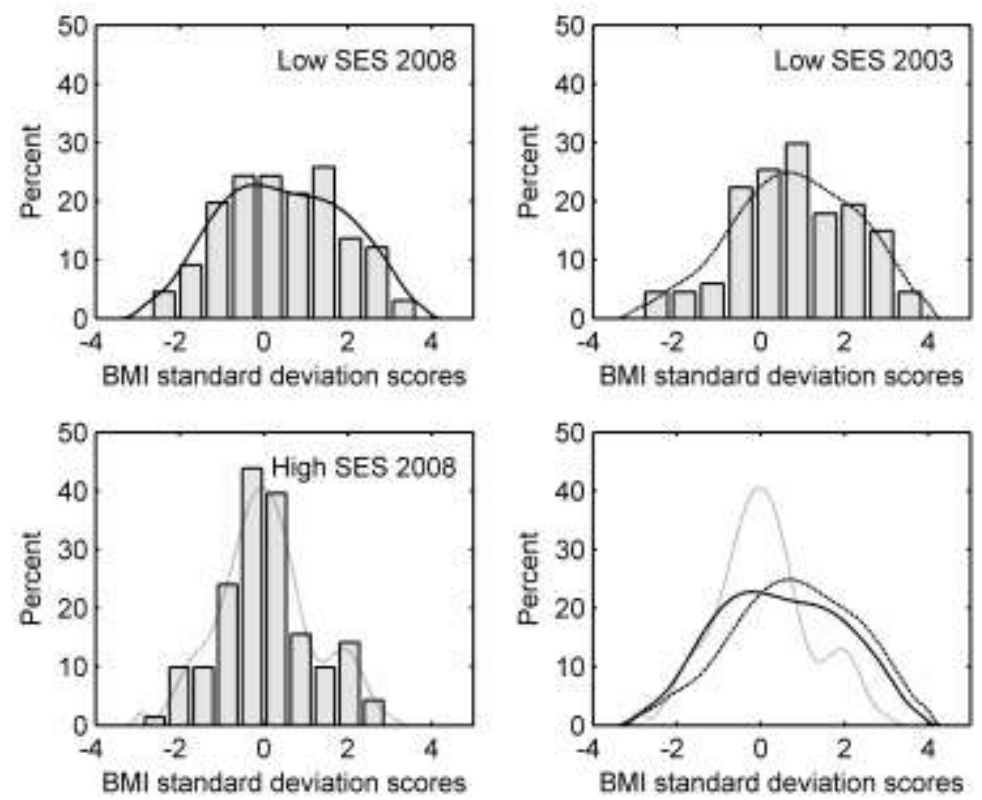

Low SES 2008

Low SES 2003 - - - High SES 2008

Figure 2 Histograms and fitted distributions of BMI z-score by school and year

Histograms and fitted distributions of BMI z-score in the low SES school 2008 and 2003, and the high SES school 2008. To facilitate a comparison between the histograms, smooth probability density estimates were overlaid. For this calculation and graphic presentation we used Matlab 7.8.0 (R2009a). The overlaid distributions (bottom right) show a shift to the left in the low SES school (dark solid and dotted lines) and, in the high SES school (light lines), a distribution which more closely corresponds to the one of the reference population.

Due to the finite bandwidth, the probability density functions extend below or above the observed values of BMI z-score, given by -2.9 and 3.9 respectively. 
Table 2 Summary of demographic and anthropometric variables in three surveys.

The surveys included in the study were conducted in Gothenburg, Sweden in 2003 and 2008. Height, weight, BMI and BMI z-score were tested by ANOVA. Obesity, overweight and thinness were tested by chi square tests.

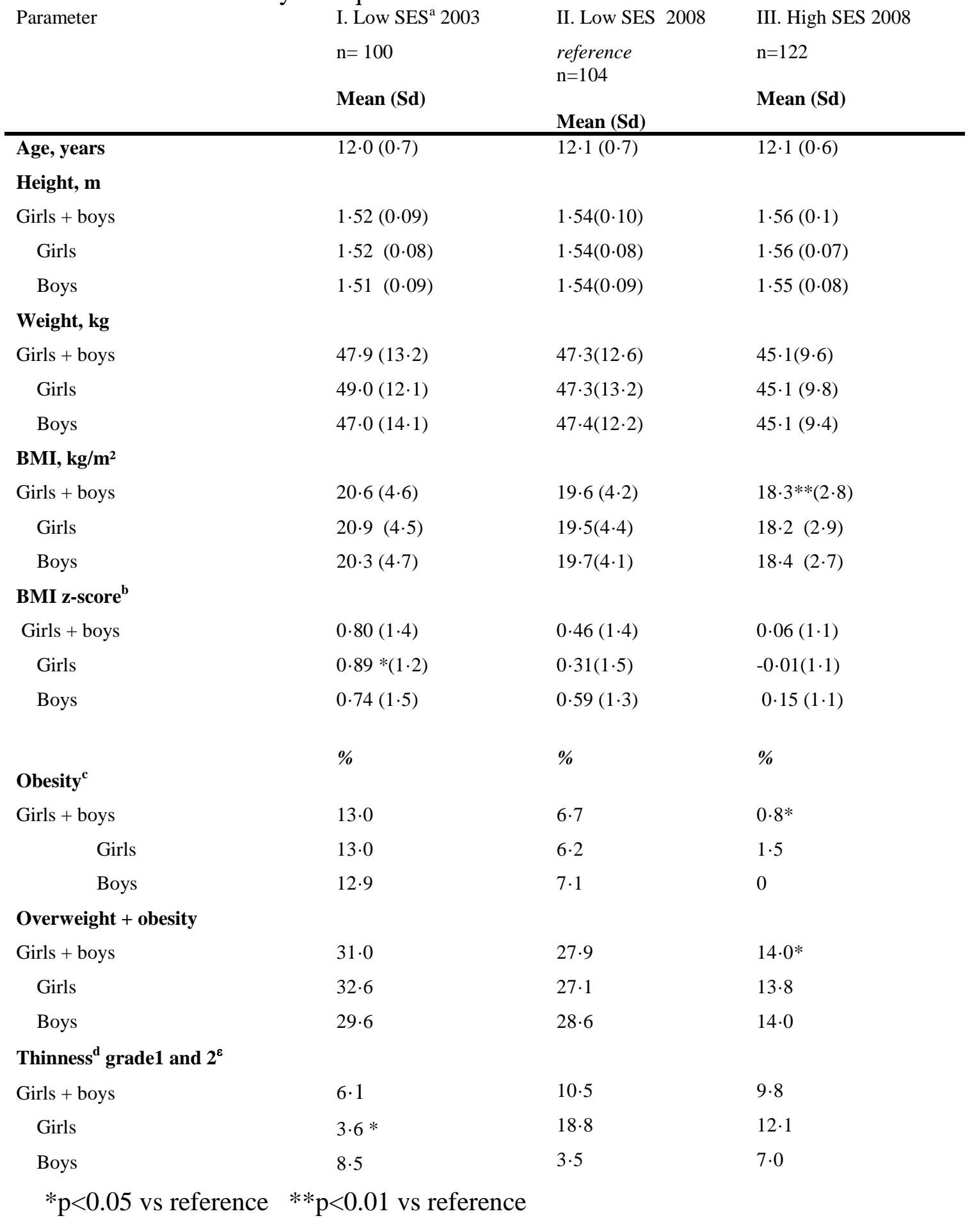

\footnotetext{
${ }^{\text {a }}$ SES: socioeconomic status

${ }^{\mathrm{b}}$ BMI z-score is calculated by the equation of Karlberg et al [16]

${ }^{\mathrm{c}}$ Obesity and overweight as defined by Cole et al [14]

${ }^{\mathrm{d}}$ Thinness as defined by Cole et al [15]

${ }^{\varepsilon}$ Out of these, one girl in 2003, one girl in low SES area in 2008 and one boy and two girls in high SES area had thinness grade 2 .
} 


\section{LIFESTYLE TRENDS IN LOW SES SCHOOL (2003 versus 2008)}

The consumption of sweet drinks, sweets and fatty snacks was significantly lower in 2008 than in 2003. More children consumed vegetables with their school lunch at least three days a week and consumed fruit daily. Fewer children had free access to sweets (defined as " "mixed candy" and such') at home in 2008 than in 2003. In contrast to these improvements, the participation in organised leisure time physical activity was significantly lower in 2008 than in 2003. Fewer children regularly played outside at the latter time-point, although this difference was not significant. No differences were observed between obese and non-obese children regarding any dietary or PA habits studied.

In 2008, significantly more boys perceived benefit of a healthy lifestyle, consumed more than seven fruits a week, habitually consumed vegetables with their school lunch and watched TV more than 2 hours a day. Significantly fewer girls had sweet drinks often, consumed sweets or fatty snacks after school and/or had sweets available daily at home (Table 3). 
Table 3 Food- and activity related life-style factors, all and by gender

The surveys included in the study were conducted in Gothenburg, Sweden in 2003 and 2008.

Tests were made by Fisher's exact chi square.

Parameter

I. Low SES ${ }^{\mathrm{a}}$

2003

II. Low

III. High SES

Parameter

$$
\mathrm{n}=108
$$

2008

$\mathrm{n}=97$

$\mathrm{n}=121$

(reference)

All
Perceiving benefit of a healthy lifestyle
Breakfast every school-day
Breakfast containing foods rich in carbohydrates, iron,
$\quad$ calcium, ascorbic acid

Having dinner daily

Having lunch in school every school day

Having vegetables for school-lunch at least 3 days/week

Sweet drinks $\geq 4$ days a week

Sweet drinks daily

Usually consumes sweets or fatty snacks in the afternoon after school

Having more than $400 \mathrm{~g}$ sweets a week

Sweets available daily at home

$\%$

$37 * *$
$50 \cdot 0$
$8 \cdot 3$

$81 \cdot 4$

$84 \cdot 2$

$52 \cdot 0^{*}$

$43 \cdot 5^{*}$

$15 \cdot 7$

Eating more than seven fruits a week

Watching television more than 2 hours a day

Using computer more than 2 hours daily, in leisure time

Playing outside several days a week

Participating in organised exercise in leisure time

Participating in organised exercise in leisure time and/or playing outside several days a week

\section{Girls/Boys}

Perceiving benefit of a healthy lifestyle

Breakfast every school-day

$19 \cdot 4^{*}$

$12 \cdot 0$

$38.0^{*}$

$25 \cdot 7 *$

$49.0 * * *$

$9 \cdot 2$

$46 \cdot 2$

$66 \cdot 7 *$

$78 \cdot 7$

Breakfast containing foods rich in carbohydrates, iron, calcium, ascorbic acid

Having dinner daily

Having lunch in school every school day

Having vegetables for school-lunch at least 3 days/week

Sweet drinks $\geq 4$ days a week

Sweet drinks daily

Usually consumes sweets or fatty snacks in the afternoon after school

Having more than $400 \mathrm{~g}$ sweets a week

Sweets available daily at home

Eating more than seven fruits a week

Watching television more than 2 hours a day

Using computer more than 2 hours daily, in leisure time

Playing outside several days a week

Participating in organised exercise in leisure time

$\mathrm{n}=\mathbf{5 3 / 5 5}$

$66 \cdot 0 / 75 \cdot 5 * *$

$41 \cdot 5 / 59 \cdot 2$

$9 \cdot 4 / 7 \cdot 4$

55

$62 \cdot 9$

$7 \cdot 2$

$\%$

$\%$

$87 * * *$

$88 \cdot 5^{* * *}$

$46 \cdot 0^{* * *}$

$86 \cdot 0$

$88 \cdot 4$

$76 \cdot 0$

$\begin{array}{ll}79 \cdot 3 & 76 \cdot 0 \\ 69 \cdot 0 & 82 \cdot 8^{*}\end{array}$

$26.8 \quad 2.5 * * *$

$7.2 \quad 0.8^{*}$

$8.2 \quad 01 *$

$79 \cdot 2 / 83 \cdot 6$

$81 \cdot 1 / 87 \cdot 3$

$12 \cdot 3$

$4 \cdot 1^{*}$

23.0

$8 \cdot 1 * *$

$41 \cdot 2$

$41 \cdot 0$

$23 \cdot 7$

$9 \cdot 0 * *$

$16 \cdot 4$

$7 \cdot 3^{*}$

$40 \cdot 2$

$63.4 * * *$

$89.9 * * *$

$50 \cdot 0$

$95 \cdot 8^{* * *}$

$66 \cdot 0 / 38 \cdot 2^{*}$

$52 \cdot 8 * / 34 \cdot 5$

$18 \cdot 9 * / 12 \cdot 8$

$20.1 * / 18.2$

$n=44 / 53$

$\mathrm{n}=\mathbf{6 5} / \mathbf{5 6}$

$81 \cdot 8 / 94 \cdot 1$

$59 \cdot 1 / 66 \cdot 0$

$96 \cdot 9 / 85 \cdot 7$

$6 \cdot 8 / 7 \cdot 5$

$86 \cdot 1 * * * / 91 \cdot 1 * *$

$55 \cdot 3 * * * / 35 \cdot 2 * * *$

$\begin{array}{lll}15 \cdot 1 / 9 \cdot 1 & 7 \cdot 5 / 19 \cdot 1 & 1 \cdot 6 / 7 \cdot 4 \\ 49 \cdot 0 * / 29 \cdot 6 & 26 \cdot 2 / 21 \cdot 2 & 4 \cdot 6 * * / 14 \cdot 3 \\ 30 \cdot 2 / 21.4^{*} & 43 \cdot 2 / 39 \cdot 2 & 45 \cdot 4 / 35 \cdot 7 \\ 50 \cdot 1 / 47 \cdot 3^{* * *} & 34 \cdot 1 / 16 \cdot 9 & 9 \cdot 2 * * / 8 \cdot 9 \\ 5 \cdot 8 / 12 \cdot 7 & 14 \cdot 3 / 18 \cdot 8 & 6 \cdot 2 / 8 \cdot 9 \\ 32 \cdot 1 / 60 \cdot 0 & 33 \cdot 3 / 47 \cdot 2 & 66 \cdot 2 * * / 62 \cdot 5 \\ 66 \cdot 7 * / 66 \cdot 7 & 42 \cdot 8 / 72 \cdot 1 & 90 \cdot 5 * / 89 \cdot 3 * * * \\ 75 \cdot 5 / 81 \cdot 8 & 64 \cdot 3 / 81 \cdot 1 & 95 \cdot 4 * * * / 96 \cdot 4 * * *\end{array}$

$88 \cdot 4 / 84 \cdot 0 \quad 87 \cdot 7 / 89 \cdot 3$

$83 \cdot 7 / 77 \cdot 4 \quad 80 \cdot 0 / 71 \cdot 4$

$77 \cdot 3 / 62 \cdot 3 \quad 88 \cdot 0 / 78 \cdot 6$

$27 \cdot 3 / 26 \cdot 4 \quad 3 \cdot 1 * * * / 1 \cdot 8 * * *$

$4 \cdot 5 / 9 \cdot 4 \quad 1 \cdot 6 / 0^{*}$

$68 / 98 \quad 16 / 0^{*}$

Participating in organised exercise in leisure time and/or playing outside several days a week

\footnotetext{
${ }^{\mathrm{a}} \mathrm{SES}$ : socioeconomic status
} 
${ }^{*} \mathrm{p} \leq 0.05$ vs reference $* * \mathrm{p} \leq 0.01$ vs reference $* * * \mathrm{p} \leq 0.001$ vs reference

\section{ANTHROPOMETRY AND LIFESTYLE IN LOW VS HIGH SES SCHOOL 2008}

The survey in the higher income school was confirmatory of what is known about SES differences in obesity, while also providing supplementary information on lifestyle factors mirroring the differences in prevalence (Tables 2 and 3).

Lifestyle habits in the children in the two residential areas showed significant differences in many respects. In the residential area with high SES, breakfast consumption was more common and its nutritional quality was better. Eating vegetables with lunch was more widespread, while fewer children frequently consumed sweet drinks, sweets or fatty snacks in the afternoon, or consumed more than $400 \mathrm{~g}$ sweets a week. Significantly more children participated in organised exercise in their leisure time in the high SES area. The proportions of girls playing outside several days a week differed significantly between the low and high SES-area. Differences were smaller and not statistically significant among boys. Spending more than 2 hours a day watching television or using computer were both significantly more common among children in the area with low SES. Significantly more children in the high SES society believed that life-style can affect health.

\section{Discussion}

\section{SUMMARY OF RESULTS, STRENGTHS AND LIMITATIONS}

Our results suggest a break in the childhood obesity epidemic in a low SES community, coinciding with the implementation of a theoretically driven community based intervention (HEI). The results add to the understanding of how to design effective preventive interventions in such areas by highlighting that gender differences in low SES communities with many immigrants and refugees may reveal themselves differently than in the majority society. The results also underline that locally based knowledge should be a taken into account when designing and evaluating studies (since standardized instruments may fail to capture relevant data). The data for girls indicated that the decrease in BMI Z-score was paralleled by a reduced consumption of calorically sweetened drinks and confectionary. The 
lower prevalence of obesity seen in the high SES compared to the low SES school was reflected in numerous disparities in dietary habits and PA.

The strengths of this study are extremely high participation and methods that were standardized including measurement techniques, interviews and questionnaires. The validation of the original instruments, which we adapted for age and level of linguistic maturity, suggest that they were suitable for dietary assessment for adolescents (Sjoberg et al. 2003). The same investigator that conducted the study in 2003 trained the dietician who assisted in 2008 .

Several important limitations of the study are also acknowledged, including the fact that there was no low SES control school surveyed in 2003 or 2008 and it was therefore not possible to study changes in a similar community without the intervention. Moreover, we recruited a school in a high income area as a comparison group for 2008, but this area had not been previously surveyed in 2003.

\section{SECULAR TRENDS AND GENDER DIFFERENCES}

Several studies in Sweden and elsewhere have described trends that are consistent with our results in that they indicate a possible levelling out of the childhood obesity epidemic. In contrast to other recent studies describing stability or reversal in trends for Swedish children representing a range of socioeconomic groups ( Lissner et al. 2009, Sjoberg et al. 2008, Sundblom et al. 2008), the present results are from a community with low SES and many immigrants. Therefore, the finding of a reduced prevalence of obesity (albeit not significant) with a significant shift downwards in BMI z-score in girls only is rather unique.

In view of the evidence that calorically sweetened drinks are related to childhood overweight and obesity (Olsen and Heitmann 2009), it is of particular interest that the decreased BMI zscore for girls was accompanied by a significant decrease in consumption of sweet drinks. The gender differences found in this study are consistent with known male-female patterns of eating, where it tends to be more socially acceptable for males to eat more (Bock and Kanarek 1995, Macmillan et al. 2010), and with studies showing that men seek help for obesity to a 
lesser extent then do women (Bertakis and Azari 2005) while women generally seem to be more troubled by excess body weight (Macmillan et al. 2010).

It has been observed that boys are more physically active than girls (Borraccino et al. 2009, Nyberg et al. 2009) and gender-specific modelling of interventions to promote PA has been proposed (Kropski et al. 2008). In our study this gender disparity was marginal in the high SES but significant in the low SES area. However, we may have failed to document some aspects of the girls' PA in the low SES area: health workers there report that many girls claim that home duties (taking care of siblings, cleaning etc) consume much of their time (personal communication). This implies that when at home, some girls are more physically active than boys. As to the decreased PA among girls between 2003 and 2008 in the low SES area, this may have to do with violent shooting incidents that occurred there 2006 - 2007. Living in high crime areas seem to affect girl's level of PA more than boy's (Gordon-Larsen et al. 2000).

\section{SOCIOECONOMIC GRADIENT AND PUBLIC HEALTH IMPLICATIONS}

Relations between SES determinants and childhood obesity are complex and not fully understood. Suggested explanations include the relatively high energy density of cheap food (Darmon and Drewnowski 2008), food deprivation in childhood as experienced by many refugees (Olson et al. 2007), parent's equating PA to "work" and thus seeking sedentary rest and relaxation when possible (Kumanyika 2008b) and increased stress in low SES children (Lupie et al. 2001).

In the low SES-community in our study, about $1 / 4$ of the whole population were born in areas where violent conflicts recently had taken place, or were ongoing. This is likely to have an impact on the health behaviour of the children in the area by multiple mechanisms, one being that many parents and grandparents have experienced scarcity or starvation and are less likely to restrict access to calorically dense food when available (Kumanyika 2008a).

Factors contributing to the disparity in PA between children in the low and high SES schools may have to do with safety and maintenance of playgrounds and access to sports facilities. 
Options to drive children to sports activities and pay for memberships in sport clubs also differ.

Our results add to a body of research showing that childhood obesity still is related to SES, and to evidence showing that community-based multi-level actions may have an effect in promoting healthier habits and reducing BMI in children from low SES groups (Chomitz et al. 2010, de Silva-Sanigorski et al. 2010). Based on our results and on research showing that adolescents are much influenced by their peers health behaviour (Richards et al. 2009), we speculate the group-level change in perceived benefit (ie belief that one is able to exert control over one's health habits) may be a background factor for the favourable change in dietary behaviours. This line of thought implies that considering psychosocial aspects when planning interventions to prevent childhood obesity could contribute to narrowing the socioeconomic health gap. The increase in thinness in girls in the low SES-school is worrisome and underlines the importance of emphasizing a broad concept of health.

\section{CONCLUSIONS}

Between 2003 and 2008 we observed a shift downwards in the BMI z-score for the girls in the low SES school, in combination with a decrease in intake of calorically sweetened drinks and confectionary. The positive changes are suggested to be a result of interplay between general societal changes and local prevention efforts. Our results underscore the importance of continuing preventive gender-sensitive efforts to promote healthy habits and slow the childhood obesity epidemic. Low SES-areas need to be prioritized and actions should be tailored for the population. A deeper understanding of the socio-cultural context of obesity in high risk areas, as well as of gender-specific and psycho-social aspects, is urgently needed.

\section{Acknowledgements}

We thank dietician Lotta Moraeus for assisting in data collection and entering data, Valter Sundh and Kirsten Mehlig for help with statistical analyses, and Professor Lena Hulthén and Professor Anna Winkvist for valuable supervision. We are also indebted to pupils and staff, especially the school nurses, in the schools. Grant support from the Region Västra Götaland, Sweden and from the Swedish Council of Working Life and Social Research (FAS) is gratefully acknowledged. 


\section{Conflicts of interest statement}

The authors declare that there are no conflicts of interest. 